\title{
Características das ações desenvolvidas por terapeutas ocupacionais nas capitais da região sudeste*
}

\section{Characteristics of actions taken by occupational therapists in the capitals of southeast region}

\author{
Solanne Gonçalves Alves ${ }^{1}$, Grasielle Silveira Tavares Paulin ${ }^{2}$ \\ http://dx.doi.org/10.11606/issn.2238-6149.v24i1p1-8
}

\begin{abstract}
Alves SG, Paulin GST. Características das ações desenvolvidas por terapeutas ocupacionais nas capitais da região sudeste. Rev Ter Ocup Univ São Paulo. 2013 jan./abr.;24(1):1-8.

RESUMO: O estudo objetiva descrever e analisar as características das ações desenvolvidas por Terapeutas Ocupacionais (T.Os) das capitais da região sudeste. Trata-se de uma pesquisa descritivoexploratória, com 17 T.Os. Utilizou-se um questionário autoadministrado e para análise dos dados, a Análise de Discurso de matriz pêcheutiana. As formações discursivas encontradas evidenciaram que as ações realizadas por T.Os compreendem um conjunto de esforços dirigidos para os indivíduos e seu contexto tanto individual como coletivo, demonstrando a singularidade profissional nas práticas clínicas, assim como a compreensão do conceito de integralidade esteve presente nos discursos de forma distinta mostrando que as diferenças culturais, tempo de formação e aperfeiçoamento profissional interferem na compreensão do processo de cuidado em Terapia Ocupacional. Conclui-se que há necessidade de novos estudos que possam mapear e aprofundar o conhecimento sobre as ações dos T.Os, afim de aprofundar a discussão sobre esta temática.
\end{abstract}

DESCRITORES: Terapia ocupacional; Assistência à saúde.
Alves SG, Paulin GST. Characteristics of actions taken by occupational therapists in the capitals of southeast region . Rev Ter Ocup Univ São Paulo. 2013 jan./abr.;24(1):1-8.

ABSTRACT: The study aims to describe and analyze the characteristics of actions of Occupational Therapists (O.Ts) from the capitals of Southeast region. This is a descriptive and exploratory search, with 17 O.Ts It used a questionnaire selfadministered and for data analysis, discourse analysis matrix pêcheutiana. The discursive formations found evidence that the actions taken by O.Ts comprise a joint of efforts aimed at individuals and their context both individual and collective, demonstrating the uniqueness professional clinical practice, as well as understanding the concept of completeness was present in the speeches differently showing that cultural differences, length of training and professional development influence the understanding of the process of care in Occupational Therapy. We conclude that there is need for further studies to map and deepen knowledge about the actions of O.Ts in order to further discuss this issue.

KEY WORDS: Occupational therapy, Delivery of health care.

Resultado do Trabalho de Conclusão de Curso (TCC) do Curso de Terapia Ocupacional da Universidade Federal do Triângulo Mineiro (UFTM) - aprovado pelo Comitê de Ética da UFTM de acordo com o parecer $\mathrm{n}^{\circ} 1566$. Parte dos resultados foram apresentados na II Mostra Científica do Curso de Terapia Ocupacional da UFTM, 2010.

1. Terapeuta Ocupacional graduada pela Universidade Federal do Triângulo Mineiro - UFTM, Residente multiprofissional em Saúde Mental pelo Instituto de Psiquiatria da Universidade Federal do Rio de Janeiro - IPUB/UFRJ.

2 Prof ${ }^{a}$ Assistente do Curso de Terapia Ocupacional da Universidade Federal do Triângulo Mineiro - UFTM.

Endereço para correspondência: Universidade Federal do Triângulo Mineiro. Unidade Centro Educacional (CE). Curso de Terapia Ocupacional. Av. Getúlio Guaritá, s/n. $3^{\circ}$ Piso. Bairro Abadia. Uberaba, MG. CEP: 38025-440. E-mail: solannealves@yahoo.com.br 


\section{INTRODUÇÃO}

tualmente a saúde distancia-se do conceito
de ausência de doença para a concepção de
projetos de vida ${ }^{12}$, sendo que o cuidado não pode ser visto apenas como uma pequena e subordinada tarefa das práticas de saúde. A atitude "cuidadora" precisa se expandir para a totalidade das reflexões e intervenções no campo da saúde, levando-se a rever uma série de pressupostos e conceitos cristalizados ao longo da hegemonia acrítica do tratar ${ }^{5}$.

No processo de cuidar, as ações desempenhadas pelos Terapeutas Ocupacionais (T.Os) compreendem a forma como cada profissional faz uso das atividades, empregam as técnicas, modificam, personalizam e transformam a atividade em algo que motive o fazer e crie possibilidades de percepção de como os sujeitos fazem, engendrando curiosidades, interesses e prazer na construção de projetos de vida junto à clientela ${ }^{17}$.

As ações desenvolvidas por T.Os são relevantes para as novas práticas de cuidado. A formação interdisciplinar dos profissionais oferece possibilidades para o desenvolvimento de ações individuais, coletivas e sempre contextualizadas ${ }^{1}$. As concepções sobre a intervenção terapêutico-ocupacional mantém a inter-relação entre pessoa-grupo-atividade e meio ambiente (sociedade/cultura/natureza), o que propicia a conexão dos sujeitos com a vida, o ambiente, os outros e com a própria subjetividade ${ }^{13}$.

$\mathrm{Na}$ diversidade das experiências brasileiras, a prática tem mostrado, a amplitude de ações plurais e que podem apresentar uma gama de oportunidades na discussão do acesso à saúde ${ }^{29}$.

Os T.Os diferenciam-se no desenvolvimento de práticas coletivas que enfocam a atividade humana, pela capacidade de análise e adaptação das atividades, bem como pela avaliação das relações que se estabelecem a partir dela. A importância dada ao processo em detrimento do produto final é o diferencial da Terapia Ocupacional ${ }^{28}$.

Esta pesquisa buscou responder à seguinte questão: quais são as características das ações dos T.Os nas capitais do sudeste brasileiro? Assumiu o objetivo de descrever o perfil dos T.Os, verificar a percepção sobre a identidade profissional e analisar os aspectos que envolvem a prática cotidiana desses profissionais.

\section{PERCURSO METODOLÓGICO}

O estudo se configurou como pesquisa do tipo descritivo-exploratória. Para a coleta dos dados, utilizou-se um questionário semi-estruturado auto-aplicado, avaliado por juízo externo, testado em pesquisa piloto, com definição dos principais termos técnicos referenciados ao final do instrumento, evitando viés no estudo.

O questionário foi composto de perguntas com subdivisões em questões fechadas, abertas e dependentes; com conteúdo sobre dados demográficos e de identificação do perfil profissional (sexo, idade, tempo de formação, área de atuação, motivação para estar no serviço, pós-graduação); questões sobre características das ações e identidade do terapeuta ocupacional: procedimentos, estratégias e recursos utilizados no cotidiano profissional, formas de avaliação da prática, compreensão sobre a importância da Terapia Ocupacional no serviço e na equipe. O questionário foi encaminhado por email a todos os T.Os que concordaram em participar do estudo entre a primeira quinzena dos meses de maio a dezembro de 2010.

Para composição da amostra, encaminhou-se correspondência, por intermédio dos Conselhos Regionais de Fisioterapia e Terapia Ocupacional (CREFITO 2, 3 e 4), aos profissionais cadastrados. As pesquisadoras também encaminharam correspondência eletrônica para a Associação Brasileira de Terapia Ocupacional e as diversas redes virtuais de Terapia Ocupacional como "RENETO"; "CampanhaTO”; "MeninasdaTO"; "Terapeutas Ocupacionais"; "Terapia Ocupacional Libertária", "Social”, "Hospitalar”, "Espírito Santo" e "U.C.B" (Universidade Castelo Branco).

Assim, foi utilizada a técnica da bola de neve ${ }^{21}$, de forma a solicitar, aos primeiros sujeitos da pesquisa, os contatos de outros possíveis sujeitos. Outro procedimento utilizado consistiu no contato com as secretarias municipais de saúde das cidades de Belo Horizonte, São Paulo, Rio de Janeiro e Vitória. Cada T.O que concordava em participar era orientado a enviar o documento com o aceite para as pesquisadoras. Após esta etapa, recebiam o questionário online.

A pesquisa foi aprovada pelos Comitês de Ética em Pesquisa da Universidade Federal do Triângulo Mineiro (UFTM) sob o parecer n ${ }^{\circ} 1566$, da Secretaria Municipal de Saúde de Belo Horizonte sob parecer n³62169P1 e Serviço de Protocolo da Prefeitura Municipal de Vitória. Não foi possível o acesso aos dados dos profissionais da Secretaria Municipal de Saúde de São Paulo e Rio de Janeiro.

A carta-convite elaborada serviu como Termo de Consentimento Livre e Esclarecido, baseada na Resolução 196/19967.

Inicialmente, propuseram-se a participar do estudo 52 T.Os, 22 deles responderam ao questionário e 17 estavam dentro dos critérios de inclusão do estudo: ser graduado em Terapia Ocupacional, atuar em serviços nas capitais da região sudeste, não exercer atividade de docência e aceitar 
participar da pesquisa. Desta forma, o universo amostral foi composto por dezessete T.Os., identificados com a letra "s" seguida de um número variável de 1 a 17.

Os resultados obtidos para identificação do perfil dos T.Os foram analisados de forma descritiva e os resultados referentes as questões qualitativas foram analisados pela técnica de Análise de Discurso de matriz pêcheutiana ${ }^{38}$. Desta forma, as formações discursivas foram encontradas nos seguintes eixos: Identidade Profissional; Características das Ações e Integralidade no Cuidado; Aspectos da Pratica Cotidiana dos T.Os: percepções, vivências e reflexões sobre a prática profissional.

\section{RESULTADOS e DISCUSSÃO}

Neste estudo, todos os sujeitos eram do sexo feminino. A predominância do gênero feminino confirma o perfil da profissão desde seu início já descrito em estudos anteriores $^{9,11,15,16,20,42,43}$. Sendo este perfil comum desde a gênese da profissão.

Quanto à faixa etária, variou entre 25 e 65 anos. Do total, 8 trabalham em São Paulo, 6 em Belo Horizonte e 3 no Rio de Janeiro.

Em relação ao tempo de formação, observou-se que 9 T.Os apresentam no máximo oito anos de formado, tendo a possibilidade de maior contato com as mudanças dos modelos assistenciais desde a formação na graduação.

A maioria dos T.Os atuam no nível terciário e quanto a área de atuação, do total de participantes, 12 desenvolvem ações na Saúde da Pessoa com Deficiência Física. Em relação ao aperfeiçoamento profissional, 10 dos pesquisados possuíam pós-graduação (lato sensu e/ou stricto sensu); 9 estavam cursando e 3 não se especializaram. Em estudo realizado por Carvalho ${ }^{11}$ com 50 T.Os atuantes nas unidades públicas de saúde da cidade do Rio de Janeiro, a maioria também tinha pós graduação.

Sobre o questionamento da motivação para os T.Os atuarem nos serviços, 14 deles relataram trabalhar nas áreas de sua preferência. Para Cobra $^{14}$, deve-se acreditar nas aspirações, fazer sempre o melhor que se pode e deixar fluir, para isto a motivação é um fator determinante no sucesso das ações profissionais.

Em relação à análise dos dados qualitativos, os resultados encontrados originaram quatro eixos de formações discursivas discutidas abaixo:

\section{Identidade Profissional}

Os dados encontrados demonstram que a complexidade em compreender a Terapia Ocupacional não é apenas conceitual e estratégica, mas relacionada à função profissional $^{26}$. O que é perceptível no discurso abaixo:

“(...) vejo grande interface entre cada uma das áreas e nem sempre consigo diferenciar a minha atuação das outras, uma vez que muitos objetivos terapêuticos podem ser contemplados nas outras áreas. Sou cobrada em dar explicações sobre a diferença entre minha atuação e a da Psicologia (...) Pedagogia (...), e nem sempre é possível; e isso pode dar uma sensação de que a Terapia Ocupacional é substituível na sua prática com um paciente" (s.9)

A Terapia Ocupacional é uma profissão que agrega conhecimentos de várias disciplinas ${ }^{31}$, por isso, sua ampla área de atuação profissional ${ }^{23,27}$ resultou em fronteiras com outras inúmeras áreas. Tais fronteiras são espaço de convergências e disputas de conhecimento e práticas ${ }^{11}$, o que explica a crise de identidade profissional. Para alguns autores, a década de 1970 foi o período de tal crise ${ }^{17}$ e para muitos, não há consenso sobre a identidade profissional ${ }^{11,17,19}$.

Bezerra et al. ${ }^{6}$ pesquisaram 49 T.Os. de Maceió, dos quais metade da amostra apresentavam insatisfação com as condições de trabalho, apontaram que o reconhecimento do papel do T.O. seria um aspecto que poderia melhorar nas condições de trabalho e ressaltaram sentimentos de impotência e frustração devido às situações vivenciadas no cotidiano da prática. Dentre as dificuldades de trabalho, Caldeira ${ }^{9}$ encontrou: a falta de conhecimento sobre a profissão, de valorização do profissional ou das atividades desenvolvidas.

Mais da metade dos participantes da pesquisa realizado por Carvalho ${ }^{11}$ referiram ter dificuldades em explicar seu trabalho e 94\% relataram que a maioria dos usuários confundia de algum modo, a Terapia Ocupacional com outras profissões.

Para Drummond e Magalhães ${ }^{18}$, isso demonstra a necessidade dos T.Os., por meio de uma leitura crítica, ampliarem a percepção das contradições sociais e que determinam a dimensão prática da sua intervenção, pois essas também se reproduzem nos espaços institucionais onde o profissional trabalha.

Assis e Pinto ${ }^{4}$ encontraram em sua amostra que $41,1 \%$, sentem dificuldade em definir a profissão e quando o fazem $80 \%$ formulam as explicações a partir das áreas de atuação do desempenho ocupacional como objetivo. Para Caniglia ${ }^{10}$ esta é uma definição que foca o objeto da profissão, o que subsidia a diferenciação da Terapia Ocupacional com as demais profissões. Este aspecto da identidade profissional também foi observado no presente estudo, conforme o discurso abaixo: 
"O Terapeuta ocupacional é extremamente importante pois é ele quem avalia os aspectos do desempenho ocupacional do sujeito, orientando suas ações no cotidiano (...) Realiza atividades que o integre em sua comunidade, pois se utiliza da lógica do território, pois tem seu olhar voltado para a realização do fazer nos espaços reais de vida. Possibilita ao individuo o fortalecimento das redes de apoio social e sua reinserção na comunidade, trabalho e moradia" (s.3)

No discurso apresentado, observa-se que o profissional consegue trocar sua identidade, apoiando-se em uma reflexão que envolve o desempenho ocupacional e a reflexão sobre o contexto na qual o sujeito está inserido, reforçando o papel do Terapeuta Ocupacional como facilitador na inclusão social da clientela.

Cabe ressaltar que a Terapia Ocupacional poderá se consolidar cada vez mais, à medida que discute seus princípios, reflete sobre sua inserção institucional e política, no âmbito do desenvolvimento das políticas assistenciais ${ }^{25}$.

\section{Cuidado}

\section{Características das Ações e Integralidade no}

A partir desta formação discursiva encontrada, buscou-se compreender como ocorrem as práticas assistenciais, os contextos de atendimentos e como os profissionais avaliam as intervenções.

Observou-se que as práticas assistenciais mais prevalentes foram: atendimento grupal, atendimento individual e orientações a familiares e usuários dos serviços. Os principais contextos de atendimento citados pelos profissionais que participaram do estudo foram o domicílio e ambulatório, sendo o local da instituição onde trabalham o espaço mais frequente das intervenções.

Quanto às formas de avaliação, notou-se que a maioria relatou realizar reavaliações e a forma de coleta dos dados mais prevalente foi a observação direta, existindo pouca utilização de testes padronizados.

Quinze T.Os relataram basear suas ações no princípio da integralidade, visando a produção de cuidado integral à saúde. Cabe ressaltar que o termo "integralidade" pode ser compreendido como a forma de atuação profissional abrangendo as dimensões biopsicossociais do indivíduo; a garantia da continuidade da atenção nos distintos níveis de complexidade do sistema de serviços de saúde; e a articulação das ações de promoção, prevenção, cura e recuperação $(\text { p.552) })^{39}$.

Nota-se que nos resultados deste estudo o termo foi contemplado pelos sujeitos de forma mais generalizada nos discursos, sendo ressaltado apenas alguns aspectos que compõem a integralidade:

“(...) intervenção não apenas com o cliente, mas considerando toda sua história de vida e o contexto em que está inserido (dinâmica familiar, potencialidades do cliente, seus desejos e possibilidades)". (s.5)

“A hanseníase é uma doença que o paciente é diagnosticado e tratado na atenção básica. Quando este paciente tem, por exemplo, problemas sensório-motor, é encaminhado para um terapeuta ocupacional, que tanto desenvolve ações de média complexidade, quanto o referencia para a alta complexidade para, por exemplo, uma cirurgia de transferência de tendão, fazendo seu pós operatório. Neste caso, como em muitos outros, o terapeuta ocupacional é um grande ponto de ligação. Com isso promove integralidade(...)" (s.10)

“(...) o sujeito recebe avaliação integral de seu estado, trabalhamos sempre em busca do fortalecimento de redes com outros equipamentos da comunidade (...)” (s1.)

Observa-se que o entrevistado s.5 traz o foco da integralidade voltado para o atendimento ao indivíduo e os entrevistados s.10 e s.1 trazem a importância da integralidade nos diversos níveis de atenção, sendo o T.O. um dos profissionais responsáveis por realizar o elo entre os equipamentos assistenciais. Porém, nota-se a ausência nos discursos da articulação das ações de promoção, prevenção e recuperação dentro de um mesmo serviço, não havendo muitas vezes integralidade destas.

Para a Análise de Discurso, as posições que os sujeitos ocupam em uma dada formação social determinam as condições de produção discursivas, definindo o lugar por eles ocupado no discurso ${ }^{38}$. Isto pode ser percebido ao correlacionar a fala dos sujeitos com a época de sua graduação, pois se sabe que muitas diretrizes curriculares do curso de Terapia Ocupacional sofreram mudanças após o surgimento e implantação do Sistema Único de Saúde (SUS.), o que corresponde atualmente a ações de promoção, prevenção e reabilitação voltadas para atenção primária, secundária e terciária. Sendo que, anteriormente, observavase maior foco nos níveis secundário e terciário com ações específicas de reabilitação, cuja mudança de cenário, simplesmente, não garantiu a adaptação do profissional, que ainda atua muitas vezes nos moldes antigos aprendidos na sua formação.

Aspectos da prática cotidiana de T.Os: percepções e vivências

Na construção desta formação discursiva, observou- 
se como os T.Os vivem e percebem sua prática assistencial e ao definirem os objetivos de suas intervenções, 12 profissionais justificaram sua prática baseando-se nas áreas de desempenho ocupacional, prevalecendo relatos de atuação referentes principalmente as atividades da vida diária (A.V.D), atividades instrumentais da vida diária (A.I.V.D), trabalho e participação social. Notou-se que as áreas referentes ao lazer, educação, brincar, descanso e sono foram citadas com menor frequência ${ }^{3}$.

Diante das percepções dos T.Os sobre seu desempenho da sua prática no campo profissional, muitos relataram que identificam que suas ações são impactantes no cotidiano dos clientes, como observado nos discursos abaixo:

“(...) pensar estratégias para que o sujeito tenha menos prejuizo possível em seu cotidiano (...) possibilita ao individuo o fortalecimento das redes de apoio social e sua reinserção na comunidade, trabalho e moradia. (s. 3)"

“A intervenção da Terapia Ocupacional traz muitos beneficios para os individuos por se tratar de uma abordagem que busca essencialmente qualidade de vida e boa interação com o ambiente e sucesso na realização das atividades cotidianas (...)" (s.4)

Os entrevistados deixam claro o impacto de suas ações no cotidiano dos clientes, ao relatarem que ao mesmo tempo em que buscam reduzir limitações, dificuldades ou barreiras; também investigam talentos, habilidades e aptidões, favorecendo o encontro entre essas habilidades e as ocupações oferecidas no mundo contemporâneo ${ }^{2}$.

Observa-se que as falas dos sujeitos corroboram com o princípio da territorialidade no sentido de reconhecimento do ambiente, população e dinâmica social existente ${ }^{41}$. Este reconhecimento é realizado através da observação das condições criadas no espaço para a produção, circulação, comunicação, lazer e condição de vida ${ }^{36}$. Pois, o Terapeuta Ocupacional, por sua formação interdisciplinar, contribui para o reconhecimento das necessidades da comunidade e dos indivíduos que nela residem, desenvolvendo, assim, atividades voltadas para a (re) inserção dos sujeitos na sociedade $^{37}$.

\section{Reflexões sobre a prática profissional}

Um aspecto importante que envolve a prática profissional está relacionado à forma como os profissionais percebem sua atuação. Apoiando-se na Análise de Discurso, é possível compreender que a formação (cultural, acadêmica, social, histórica) de cada Terapeuta Ocupacional influenciara na forma de ver e fazer Terapia Ocupacional, trazendo ações plurais e valorização de determinados aspectos em detrimento de outros. Nos discursos, notouse que muitas vezes as ações promotoras de saúde, como orientação aos familiares, deixaram de ser valorizadas trazendo a centralidade da atenção somente para os procedimentos que envolvem o indivíduo e podem obter resultados mais concretos. Estes aspectos são notados nos discursos abaixo:

"adolescentes e adultos com Paralisia Cerebral, em que as deformidades já estão instaladas de maneira permanente, vejo minha atuação com baixo nivel de evolução, somente preventiva, de manutenção. Com as crianças do setor de Intervenção Precoce, há maiores possibilidades de ganhos motores e cognitivos, e busca de independência nas AVDs. Com as crianças autistas e psicóticas, podem ser traçados muitos objetivos terapêuticos, (...)" (s.9)

\begin{abstract}
"Muitas vezes não conseguimos corresponder às expectativas que elas possuem, pois não se encaixam com seus prognóstico e evolução do quadro, por isso muitos não ficam satisfeitos. Outros pacientes conseguem perceber que os pequenos ganhos já são muito consideráveis." (s1)
\end{abstract}

Observa-se na fala do entrevistado s.9 que há um reconhecimento da realização de ações preventivas e de manutenção, todavia nota-se valorização das ações reabilitadoras. É importante frisar que a atuação do Terapeuta Ocupacional não se limita a assistir o individuo, mas também sua família e comunidade a qual ele pertence, fazendo com que as ações de promoção de saúde, prevenção de doenças e recuperação atuem de forma dinâmica. Uma reflexão importante pode ser feita frente a maioria dos participantes do estudo atuarem na atenção terciaria à saúde, o que contribui para prevalência e valorização das ações reabilitadoras, necessitando, desta forma, de um diálogo entre os diversos níveis de atenção. Sendo assim, compartilha-se do questionamento trazido por Galheigo ( p. 114$)^{22}$ :

Afinal, que conhecimentos a terapia ocupacional incorpora neste diálogo? (...) segue a racionalidade da especialidade médica ou a da saúde coletiva? Mistura ambas? Organiza sua intervenção pelo enfoque da reabilitação ou busca também incorporar a concepção da promoção da saúde? Como entende os processos saúde-doença: pela perspectiva naturalizante da doença ou (...) enquanto produção social? ${ }^{22}$. 
Alves SG, Paulin GST. Características das ações. Rev Ter Ocup Univ São Paulo. 2003 jan./abr.;24(1):1-8..

Ainda que o modelo de tratar e prevenir doenças começara a ser questionado a partir dos anos 1960, pela percepção que os fatores chaves para a melhoria da saúde eram sócioambientais e não médicos ${ }^{40}$, reconhece-se que tal modelo ainda exerce influências nas práticas de saúde. Percebe-se nas formações discursivas dos T.Os que há valorização da técnica em detrimento das relações - tecnologias leves ${ }^{33-35}$. Sabe-se mais sobre o particular sem, contudo, avançar no sentido de um entendimento abrangente dos fenômenos humanos ${ }^{32}$. O problema é a falta de reflexão crítica, na qual se trata a patologia ao sujeito $^{24}$. Pois, o empenho desmedido pela técnica perfeita, transforma o cliente em objeto ${ }^{30}$.

Percebe-se que a prática cotidiana da Terapia Ocupacional exige sensibilidade e reflexão para que o processo de cuidar possa compreender o indivíduo que se move e o profissional possa desenvolver ações que abarquem as singularidades presentes em cada fazer.

\section{CONCLUSÃO}

Em relação ao perfil profissional dos T.Os participantes do estudo, notou-se prevalência de profissionais do sexo feminino, tendo metade da amostra no máximo oito anos de formado, preferencialmente atuação em nível terciário e ações desenvolvidas na área da saúde da pessoa com deficiência física. Quanto ao aperfeiçoamento profissional, notou-se que a busca por cursos de pós-graduação é algo frequente na categoria profissional. Verificou-se também, que existe grande motivação para atuar nos serviços, sendo que a maioria relatou trabalhar na área de sua preferência.

Observou-se que as praticas assistenciais mais prevalentes foram: atendimento grupal, atendimento individual e orientações a familiares e usuários dos serviços. Verificou-se que os principais contextos de atendimentos são os locais da própria instituição onde trabalham. E quanto às formas de avaliação notou-se que a maioria relatou realizar reavaliações e a forma de coleta dos dados mais prevalente foi a observação direta, existindo pouca utilização de testes padronizados.

Constatou-se que apesar de alguns profissionais demonstrarem-se seguros e fundamentados quanto sua atuação, alguns apresentaram crise na identidade profissional e demonstraram estar em processo de adaptação às novas tendências em saúde. No entanto, observou-se que muitos conseguem ajustar sua prática aos moldes dos serviços atuais.

Um dos grandes desafios desta pesquisa foi conseguir uma amostra significativa, frente a isto os dados não podem ser generalizados e alertam para a necessidade de novos estudos para compreender melhor a problemática e da conscientização dos T.Os quanto à importância de participarem das pesquisas afim de divulgar as práticas assistenciais e conquistar novos espaços de atuação.

Agradecimentos: Agradecemos às Secretarias Municipais de Saúde dos municípios de Belo Horizonte e Vitória (Serviço de Protocolo), pela viabilidade de realização desta pesquisa e à professora Lucia Uchoa Figueiredo, pela avaliação do questionário.

\section{REFERÊNCIAS}

1. Almeida MC, Oliver FC. Abordagens comunitárias e territoriais em reabilitação de pessoas com deficiências: fundamentos para a terapia ocupacional. In: De Carlo MMRP, Bartalotti CC, organizadores. Terapia ocupacional no Brasil: fundamentos e perspectivas. 2a ed. São Paulo: Plexus; 2002. p.81-98.

2. Almeida DT, Trevisan ER. Estratégias de intervenção da terapia ocupacional em consonância com as transformações da assistência em saúde mental no Brasil. Interface (Botucatu). 2011;15(36):299-307.
3. AOTA. Occupational therapy practice. Am J Occup Ther. 2008;63(6):625-8.

4. Assis CP, Pinto MPP. Dificuldades encontradas por estudantes e profissionais do município de Uberaba ao explicarem a Terapia Ocupacional. Cad Ter Ocup UFSCar (São Carlos). 2010 set./dez. 18(3):263-74.

5. Ayres JRCM. Tão longe tão perto: o cuidado como desafio para pensar e fazer nas práticas de saúde. In: VII Encontro de Pesquisadores em Saúde Mental, VI Encontro de 
Especialistas em Enfermagem Psiquiátrica, Ribeirão Preto; 2002. Comunicação oral. [Datilogr.].

6. Bezerra WC, Tavares MMF, Cavalcante GMM. O mercado de trabalho da terapia ocupacional em Maceió-AL no contexto contemporâneo de crise do capital. Rev Ter Ocup Univ São Paulo. 2009 maio/ago.;20(2):75-84.

7. Brasil. Ministério da Saúde. Conselho Nacional de Saúde. Resolução 196/1996. Diretrizes e normas regulamentadoras de pesquisas envolvendo seres humanos. Brasília: Ministério da Saúde; 1996.

8. Brito CMD. Formação do Terapeuta Ocupacional no século XXI. Rev Baiana Saude Publ. 2008;32(supl. 1):29-39.

9. Caldeira VA. Prática de terapia ocupacional em Unidade de Saúde na atenção às pessoas com deficiência [Dissertação]. São Paulo: Universidade de São Paulo, 2009.

10. Caniglia MM. Terapia ocupacional: um enfoque disciplinar. Belo Horizonte: Ophicina de Arte e Prosa; 2005.

11. Carvalho CRA. A atuação dos terapeutas ocupacionais em unidades públicas de saúde da Cidade do Rio de Janeiro [Dissertação]. Rio de Janeiro: Escola Nacional de Saúde Pública Sergio Arouca, Fundação Oswaldo Cruz, Ministério da Saúde; 2010.

12. Castro ED, Silva DM. Habitando os campos da arte e da terapia ocupacional: percursos teóricos e reflexões. Rev Ter Ocup Univ São Paulo. 2002;13(1):1-8.

13. Castro ED, Silva DM. Atos e fatos de cultura: territórios das práticas, interdisciplinaridade e as ações na interface da arte e promoção da saúde. Rev Ter Ocup Univ São Paulo. 2007;18(3):102-12.

14. Cobra N. A semente da vitória. São Paulo: Senac; 2001.

15. Craick C, Zaccaria J. The carrer choice of first-year occupational therapy students: A follow-up study. Br J Occup Ther. 2003;66(11):531-5.

16. Craick C, Wyatt-Rolason T. Characteristics of students who enter occupational therapy through the universities and colleges admissions service (UCAS) clearing system. Br J Occup Ther. 2002;6(11):488-94.

17. De Carlo MMRP, Bartolotti CC. Terapia ocupacional no Brasil, fundamentos e perspectivas. São Paulo: Plexus Editora; 2001.

18. Drumond AF, Magalhães LC. Tendências da formação do terapeuta ocupacional no Brasil. Rev Ter Ocup Univ São Paulo. 2001;12(1):34-9.

19. Farinha AC, Silva CV. Da consciência individual à identidade colectiva - um contributo para a compreensão da identidade profissional dos terapeutas ocupacionais portugueses. In: Re (habilitar) Rev. ESSA. 2005;(1)::101-29. Disponível em: http://www.essa.pt/revista/docs/n_1/Re_habilitar_1_5.pdf

20. Fleming J, Gilbert J, Mckena K, Health T. First year occupational therapy students: profile and perceptions. Austr Occup Ther J. 1997;44:107-18.

21. Flick UWE. Introdução à pesquisa qualitativa; tradução Joice Elias Costa- 3. ed. Porto Alegre: Artmed, 2009.

22. Galheigo SM. Domínios e temáticas no campo das práticas hospitalares em terapia ocupacional: uma revisão da literatura brasileira de 1990 a 2006. Rev Ter Ocup Univ São Paulo. 2007;18(3):113-21.

23. Galheigo SM. Transdiciplinaridade enquanto princípio e realidade das ações de saúde. Rev Ter Ocup Univ São Paulo. $1999 ; 10(2 / 3): 49-54$.

24. Gallian DMC. A (re) humanização da medicina. Psiq Prat Med. 2000;33(2):5-8.

25. Jardim TA, Afonso VC, Pires IC. A terapia ocupacional na estratégia de saúde da família - evidências de um estudo de caso no município de São Paulo. Rev Ter Ocup Univ São Paulo,. 2008;19(3):167-75.

26. Liberman F, Tedesco S, Samea M. Habilitando a reabilitação - as ações da Terapia Ocupacional em sua integralidade: a necessidade de discussão conceitual sobre os processos de reabilitação. Rev Mundo Saúde (São Paulo). 2006;30:146-50.

27. Lima EMPA. Identidade e complexidade: composições no campo da terapia ocupacional. Rev Ter Ocup Univ São Paulo. 1999;10(2/3):42-6.

28. Lopes RE, Leão A. Terapeutas ocupacionais e os centros de convivência e cooperativa: novas ações de saúde. Rev Ter Ocup Univ São Paulo. 2002;13(2):56-63.

29. Malfitano APS, Lopes RE. Programa de saúde da família e agentes comunitários: demandas para além da saúde básica. Rev.Ter Ocup Univ São Paulo. 2003;14(3):110-7.

30. Martim LM. A ética e a humanização hospitalar. Mundo Saúde (São Paulo). 2003;27:206-18.

31. Medeiros MHRA. A terapia ocupacional em relação à 
Alves SG, Paulin GST. Características das ações. Rev Ter Ocup Univ São Paulo. 2003 jan./abr.;24(1):1-8..

produção de conhecimento. Cad Ter Ocup UFSCAR (São Carlos). 2003;8(1):61-8.

32. Medina JPS. O brasileiro e seu corpo. 3a ed. Campinas: Papirus; 1991.

33. Merhy EE. A perda da dimensão cuidadora na produção da saúde - Uma discussão do modelo assistencial e da intervenção no seu modo de trabalhar a assistência. In: Malta DC, et al. Sistema Único de Saúde em Belo Horizonte reescrevendo o público. Belo Horizonte: Xamã/VM Ed.; 1998.

34. Merhy EE. Saúde: cartografia do trabalho vivo. 3a ed. São Paulo: Hucitec; 2002.

35. Merhy EE. O ato de cuidar: a alma dos serviços de saúde. In: Brasil. Ministério da Saúde. Secretaria de Gestão do Trabalho e da Educação na Saúde. Departamento de Gestão da Educação na Saúde. Ver - SUS Brasil: cadernos de textos. Brasília: Ministério da Saúde; 2004. p.108-137. (Série B. Textos Básicos de Saúde).

36. Monken M, Barcellos C. Vigilância em saúde e território utilizado: possibilidades teóricas e metodológicas. Cad Saúde Pública (Rio de Janeiro). 2005;21(3):898-906. Disponível em: http://dx.doi.org/10.1590/S0102-311X2005000300024.

37. Oliver FC, Almeida MC. Reabilitação baseada na comunidade. In: Cavalcanti A,, Galvão C. Terapia ocupacional fundamentação e prática. Rio de Janeiro: Guanabara Koogan; 2007. p.125-32.

38. Orlandi EP. Análise do discurso: princípios e procedimentos. 8a ed. Campinas: Pontes; 2009.

39. Paim JS. Modelos de atenção à saúde no Brasil. In: Giovanella L, et al. Políticas e sistema de saúde no Brasil. Rio de Janeiro: Editora Fiocuz; 2008. p.547-46.

40. Pereira AC. Odontologia em saúde coletiva. Porto Alegre: Artmed; 2003.

41. Pereira MP, Barcellos C. O território no Programa Saúde da Família. Hygeia Rev Bras Geogr Med Saúde (Uberlândia). 2006;2(2):47-55.

42. Pfeifer LI. Análise de concepções e expectativas de alunos recém-ingressos no curso com relação ao mesmo e a profissão. Cad Ter Ocup UFSCar (São Carlos). 1996;5(2):101-10.

43. Silva AFF, Silva CV. Perfil dos estudantes de terapia ocupacional da Escola Superior de Saúde do Alcoitão. Re (habilitar) Rev ESSA Esc Sup Saúde Alcoitão (Alcabideche, Portugal,). 2006;3:39-67 [citado 20 nov. 2010]. Disponível em: http://www.essa.pt/revista/docs/n_3/Re_habilitar_3_3. pdf. 